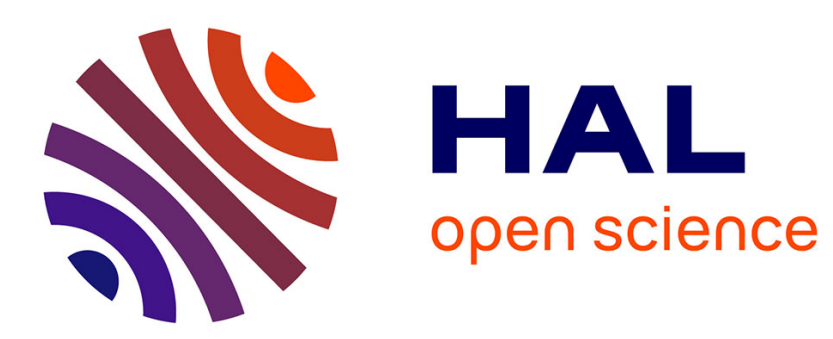

\title{
Effects of order on the electronic structure of ferromagnetic transition metal alloys: application to FeCo and $\mathrm{Ni} \_3 \mathrm{Fe}$
}

M. C. Desjonquères, M. Lavagna

\section{- To cite this version:}

M. C. Desjonquères, M. Lavagna. Effects of order on the electronic structure of ferromagnetic transition metal alloys: application to FeCo and Ni_3Fe. Journal of Physics F: Metal Physics, 1979, 9 (9), pp.1733 - 1743. 10.1088/0305-4608/9/9/005 . hal-01896487

\section{HAL Id: hal-01896487 \\ https://hal.science/hal-01896487}

Submitted on 16 Oct 2018

HAL is a multi-disciplinary open access archive for the deposit and dissemination of scientific research documents, whether they are published or not. The documents may come from teaching and research institutions in France or abroad, or from public or private research centers.
L'archive ouverte pluridisciplinaire HAL, est destinée au dépôt et à la diffusion de documents scientifiques de niveau recherche, publiés ou non, émanant des établissements d'enseignement et de recherche français ou étrangers, des laboratoires publics ou privés. 


\title{
Effects of order on the electronic structure of ferromagnetic transition metal alloys: application to $\mathrm{FeCo}$ and $\mathrm{Ni}_{3} \mathrm{Fe}$
}

\author{
M C Desjonquères† and $\mathrm{M}$ Lavagna \\ + Groupe des Transitions de Phases, CNRS, 166X, 38042 Grenoble-Cédex, France and \\ Laboratoire de Microscopie Ionique, Faculté des Sciences et Techniques de Rouen 76 \\ 130-Mont-Saint-Aignan, France \\ † Laboratoire Louis Néel, CNRS, 166X, 38042 Grenoble-Cédex, France
}

Received 6 March 1979

\begin{abstract}
The electronic structures of ordered and disordered transition metal alloys are described in a tight-binding scheme. Charge transfer and magnetism are treated within a self-consistent Hartree-Fock model. We show that the rigid band splitting fails to take account of the ferromagnetism of these compounds. The densities of states of FeCo and $\mathrm{Ni}_{3} \mathrm{Fe}$ are found to be more sensitive to order than the ferromagnetic properties. Energetic calculations are performed giving an estimation of the ordering energy, and comparisons are made with experimental results.
\end{abstract}

\section{Introduction}

Recently there has been much progress in our knowledge of the properties of binary transition alloys. Yet they are far from being fully understood. It is the $\mathrm{FeCo}$ and $\mathrm{NiFe}$ alloys which are particularly interesting as, below some characteristic temperature, they can make ordered structures when they are of well defined composition and they can be ferromagnetic. So for instance the investigation of the influence of order on the electronic and magnetic properties is a very difficult task. Indeed, from a theoretical point of view, the rigid splitting of the up and down spin bands fails for the description of alloys as we will emphasise. The difficulties of treating the alloy problem and the ferromagnetism in a realistic manner are then added. It is the purpose of this paper to show that this is nevertheless possible in the ordered as well as in the disordered states, and to derive an estimation of ordering energies which, up to now, has been carried out only in the case of non-magnetic alloys (Giner et al 1976, Ducastelle and Gautier 1976).

We will here consider the case of equiconcentrational $\mathrm{BCC}$ and $\mathrm{FCC} F \mathrm{FeCo}$ and $\mathrm{FCC} \mathrm{Ni}_{3} \mathrm{Fe}$ alloys. The evolution of their properties with temperature is given in figure 1 . The electronic structure of binary transition alloys is usually investigated in the framework of the tight-binding approximation. Yamashita et al (1966a, b) have studied the case of ordered $\mathrm{FeCo}$ and $\mathrm{Ni}_{3} \mathrm{Fe}$ alloys but with no attempts at a self-consistent determination of the difference between the effective atomic levels of each 

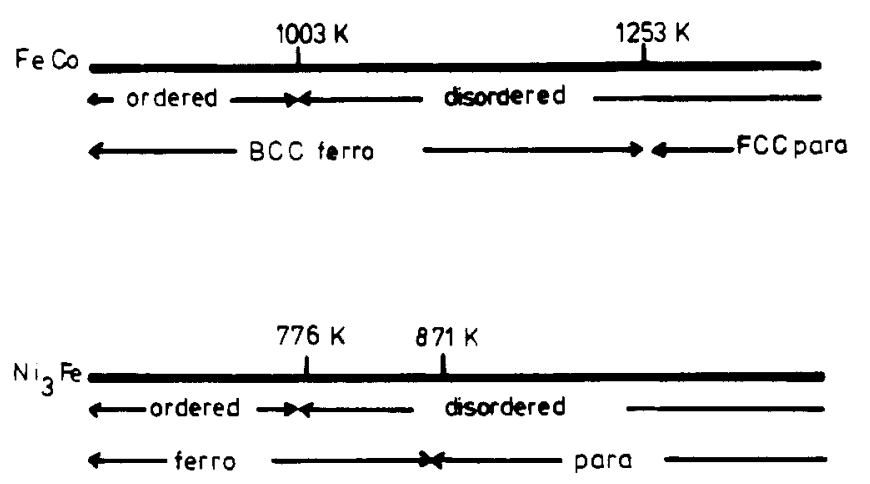

Figure 1. Phase stability of $\mathrm{FeCO}$ and $\mathrm{Ni}_{3} \mathrm{Fe}$ with temperature.

species. The case of ferromagnetic disordered FeCo and NiFe alloys has been considered by Hasegawa and Kanamori (1971, 1972a, b) and Jo (1976) in the CPA approximation, taking into account the self-consistency problem, but in a single-band model. Moreover, they used a schematic model for the density of states of the pure metals and they completely neglected off-diagonal disorder which has certainly an important effect, especially for NiFe alloys. No comparison of the ordered and disordered alloys has been performed and, consequently, there is not yet any theoretical estimation of the ordering energy derived from electronic structure calculations. Here, contrary to previous works, we will take into account the degeneracy of the $\mathrm{d}$ band, the charge transfer and ferromagnetism in a Hartree-Fock self-consistent scheme and the effect of off-diagonal disorder.

In $\$ 2$ we will outline the methods of calculation (moment method for ordered alloys, generalisation of the coherent potential approximation (CPA) to off-diagonal disorder (Shiba 1971)) and we will discuss in more details the self-consistency problem. In $\S 3$, we will compare the density of states of $\mathrm{FeCo}$ and $\mathrm{Ni}_{3} \mathrm{Fe}$ in the ordered and disordered states and their magnetic behaviour. We will show that the density of states is more sensitive to order than the ferromagnetic properties. For each state of the alloys, we will compute its energy per atom and the values of those energies will be found to explain fairly well the evolution of their properties with temperature. We will also give the first theoretical estimation of the ordering energies derived from electronic structure. Conclusions will be drawn in $\$ 4$.

\section{Method}

The $\mathrm{d}$ band of transition metals or alloys is well described by the tight-binding Hamiltonian:

$$
H^{\sigma}=\sum_{i j} E_{i \sigma}|i \lambda \sigma\rangle\left\langle i \lambda \sigma\left|+\sum_{\substack{i j \\ i \mu}} \beta_{\lambda \mu}^{i j}\right| i \lambda \sigma\right\rangle\langle j \mu \sigma|
$$

where $|i \lambda \sigma\rangle$ is the atomic orbital centred on site $i$, with symmetry $\lambda(x y, y z, z x$, $x^{2}-y^{2}, 3 z^{2}-r^{2}$ ), and $\sigma$ is the spin. The $\beta_{\lambda, k}^{i j}$ are the two-centre hopping integrals between first-nearest neighbours for FCC crystals and also second-nearest neighbours for BCC crystals. They can be expressed in terms of three parameters $\mathrm{dd} \sigma, \mathrm{dd} \pi$, $\mathrm{dd} \delta$ and the direction cosines of the bond (Slater and Koster 1954). For an AB alloy, 
these parameters can take three values according to the occupancies of sites $i$ and $j$. When the atoms at sites $i$ and $j$ are identical, they are derived from a fit of bandstructure calculations, and they are eventually corrected for the distance $\mathrm{d}$-with a $\mathrm{d}^{-5}$ law-as the interatomic spacing can be different in the alloy and in the pure metal. When the hopping occurs between different atoms, we will estimate the parameters using Shiba's formula (Shiba 1971), i.e. as a geometrical average of the parameters relative to the pure metals.

$E_{i \sigma}$ is the effective atomic level on site $i$; it depends on the nature of the atom at site $i$ but also on the occupation numbers of the various $|i \lambda \sigma\rangle$ orbitals. Its determination then requires a self-consistent procedure that we will explain below in more detail.

For ordered alloys, $\beta_{i \mu}^{i j}$ and $E_{i \sigma}$ being known, the local density of states (LDS) on site $i$ and spin $\sigma$ is given by:

$$
n_{i}(E)=\sum_{\lambda, n} \mid\left\langle n\left|i \lambda_{n}\right|^{2} \delta\left(E-E_{n}\right)\right.
$$

where $|n\rangle$ is the eigenfunction belonging to the eigenvalue $E_{n}$. It is easily computed in terms of its first moments, using the continued fraction technique (Gaspard and Cyrot-Lackmann 1973, Desjonquères and Cyrot-Lackmann 1975). The total density of states of the ordered alloy is:

$$
n(E)=c n_{\mathrm{A}}(E)+(1-c) n_{\mathrm{B}}(E)
$$

where $c$ and $(1-c)$ are respectively the concentrations in the $\mathrm{A}$ and $\mathrm{B}$ components.

In the case of disordered alloys, the moment method can also in principle be used but, as $E_{i \sigma}$ and $\beta_{i \mu}^{i j}$ are random, averaging over many configurations is needed. This process has been used for narrow $s$ band alloys (Desjonquères and Cyrot-Lackmann 1977) and has shown that, when the atomic levels are not too different and for nearly equiconcentrational alloys, CPA gives quite resonable results. We will then use the generalisation of CPA to off-diagonal disorder (Shiba 1971). The self-energy $\Sigma(z)$ is the solution of:

$1=\frac{c}{1+\left[\mathscr{L}_{\mathrm{A}}^{-1}-(z-\Sigma)\right] F(z-\Sigma)}+\frac{1-c}{1+\left[\mathscr{L}_{\mathrm{B}}^{-1}-(z-\Sigma)\right] F(z-\Sigma)}$

where $\mathscr{L}_{\mathrm{A}}^{-1}$ and $\mathscr{L}_{\mathrm{B}}^{-1}$ are the renormalised Shiba's locators

$$
\mathscr{L}_{\mathrm{A}(\mathrm{B})}^{-1}=\frac{Z-E_{\mathrm{A}(\mathrm{B})}}{W_{\mathrm{A}(\mathrm{B})}}
$$

$E_{\mathrm{A}(\mathrm{B})}$ being the effective atomic level of $\mathrm{A}(\mathrm{B})$ atoms, and $W_{\mathrm{A}(\mathrm{B})}$ the bandwidth of the pure metal $\mathrm{A}(\mathrm{B}) . F(z)$ is the Green's function of a pure metal of unit bandwidth, calculated with the moment method. Then

$$
n_{\mathrm{A}(\mathrm{B})}(E)=-\frac{1}{\pi W_{\mathrm{A}(\mathrm{B})}} \operatorname{Im} \frac{F(z-\Sigma)}{1+\left[\mathscr{L}_{\mathrm{A}(\mathrm{B})}^{-1}-(z-\Sigma)\right] F(z-\Sigma)}
$$

and (3) gives the total density of states.

Within the Hartree-Fock approximation, in the ordered as well as in the disordered case, the effective atomic levels are given by

$$
\begin{aligned}
& E_{\mathrm{A} \uparrow}=E_{\mathrm{A}}^{0}+\left(9 U_{\mathrm{A}}-4 J_{\mathrm{A}}\right) \Delta Z_{\mathrm{A}} / 10-\left(U_{\mathrm{A}}+4 J_{\mathrm{A}}\right) M_{\mathrm{A}} / 10 \\
& E_{\mathrm{A} \downarrow}=E_{\mathrm{A}}^{0}+\left(9 U_{\mathrm{A}}-4 J_{\mathrm{A}}\right) \Delta Z_{\mathrm{A}} / 10+\left(U_{\mathrm{A}}+4 J_{\mathrm{A}}\right) M_{\mathrm{A}} / 10
\end{aligned}
$$


where $\Delta Z_{\mathrm{A}}$ is the charge transfer on atom $\mathrm{A}$ and $M_{\mathrm{A}}$ is its magnetic moment in Bohr magnetons. $U_{\mathrm{A}}, J_{\mathrm{A}}$ are the Coulomb and exchange integrals of metal A. Similar equations are valid for $\mathrm{B}$ atoms. The energy difference $\left(E_{\mathrm{A}}^{0}-E_{\mathrm{B}}^{0}\right)$ is drawn from Herman and Skillman tables (Herman and Skillman 1963) assuming an $n \mathrm{~d}^{p+1}$ $(n+1) \mathrm{s}^{1}$ configuration of the free atoms $(n=3,4$ or 5$)$. We thus have to solve a self-consistent problem with three parameters: $\Delta Z_{\mathrm{A}}, M_{\mathrm{A}}, M_{\mathrm{B}}$ for instance, the fourth one $\Delta Z_{\mathrm{B}}$ being obtained from the condition of charge conservation. In the case of strong ferromagnetism, there is only one parameter as the spin-up band is filled. Starting from input values of these parameters, we calculate:

$$
\begin{aligned}
\Delta_{\uparrow} & =E_{\mathrm{A} \uparrow}-E_{\mathrm{B} \uparrow} \\
\Delta_{\downarrow} & =E_{\mathrm{A} \downarrow}-E_{\mathrm{B} \downarrow}
\end{aligned}
$$

and then $n_{\mathrm{A}}(E), n_{\mathrm{B}}(E), n(E)$ for each spin from which we derive output values of $\Delta Z_{\mathrm{A}(\mathrm{B})}, M_{\mathrm{A}(\mathrm{B})}$ which must be consistent with the input ones. We can thus derive the solution with a precision of about $10^{-3}$ electron. Let us notice that it would be incorrect to take ferromagnetism into account by a simple rigid splitting of the paramagnetic density of states. Indeed:

$$
\Delta_{*}-\Delta_{\downarrow}=-U\left(M_{\mathrm{A}}-M_{\mathrm{B}}\right) / 5
$$

(We assume here $U_{\mathrm{A}}=U_{\mathrm{B}}=U, J_{\mathrm{A}}=J_{\mathrm{B}}=0$ ) so the spin-up and spin-down bands have not the same shape especially when the local magnetic moments $M_{\mathrm{A}}$ and $M_{\mathrm{B}}$ are very different.

Calculations show that the results are quite insensitive to the values of $J_{\mathrm{A}}$ and $J_{\mathrm{B}}$ and to a small difference between $U_{\mathrm{A}}$ and $U_{\mathrm{B}}$. So we make the simplifying assumption $U_{\mathrm{A}}=U_{\mathrm{B}}=U$ and $J_{\mathrm{A}}=J_{\mathrm{B}}=0$. The energy $E_{\mathrm{AB}}$ of all the phases of the compounds can be deduced from the spin-up and spin-down densities of states by means of:

$$
\begin{gathered}
E_{\mathrm{AB}}=5\left[\int^{E_{\mathrm{F}}} E n_{\uparrow}(E) \mathrm{d} E+\int^{E_{\bar{F}}} E n_{\downarrow}(E) \mathrm{d} E-c U\left(5 N_{\mathrm{A} \uparrow} N_{\mathrm{A} \downarrow}+2 N_{\mathrm{A} \uparrow}^{2}+2 N_{\mathrm{A} \downarrow}^{2}\right)\right. \\
\left.-(1-c) U\left(5 N_{\mathrm{B} \uparrow} N_{\mathrm{B} \downarrow}+2 N_{\mathrm{B} \uparrow}^{2}+2 N_{\mathrm{B} \downarrow}^{2}\right)\right] .
\end{gathered}
$$

The last terms correspond to the Coulomb interaction which is taken into account twice in the one-electron energy (Lavagna 1978). $N_{\mathrm{A}+}\left(N_{\mathrm{A} \downarrow}\right)$ is the average number of electrons of spin-up (down) per orbital of metal A (B).

\section{Results}

\section{1. $\mathrm{FeCo}$}

We deduce the values of the hopping integrals from those given by Desjonquères and Cyrot-Lackmann (1975) in the case of BCC iron with a bandwidth of $0.43 \mathrm{Ryd}$ for $\mathrm{Fe}$ and $0.36 \mathrm{Ryd}$ for $\mathrm{Co}$. The difference $\left(E_{\mathrm{Fe}}^{0}-E_{\mathrm{C}_{0}}^{0}\right)$ between the atomic levels of the two components is taken to be 0.055 Ryd (Herman and Skillman 1963). Each atom of $\mathrm{Fe}$ and $\mathrm{Co}$ brings respectively 7 and 8.3 electrons to the $\mathrm{d}$ band. The value of the Coulomb integral is taken to be $0.33 \mathrm{Ryd}$. 
The density of states of the ferromagnetic state can then be computed selfconsistently following the method described above. In both ordered and disordered phases, we find that the compound exhibits strong ferromagnetism. Curves are given in figure 2. From a general point of view, for each spin, they are similar to the shape of the density of states of a pure BCC metal with a deep valley in the middle of the band. The majority spin bands are not very different in the ordered and disordered cases, contrary to the minority spin bands where the second peak becomes very smooth in the disordered phase. This can be explained within the rigid band model, knowing that the cobalt band is narrower than the iron one. For the minority

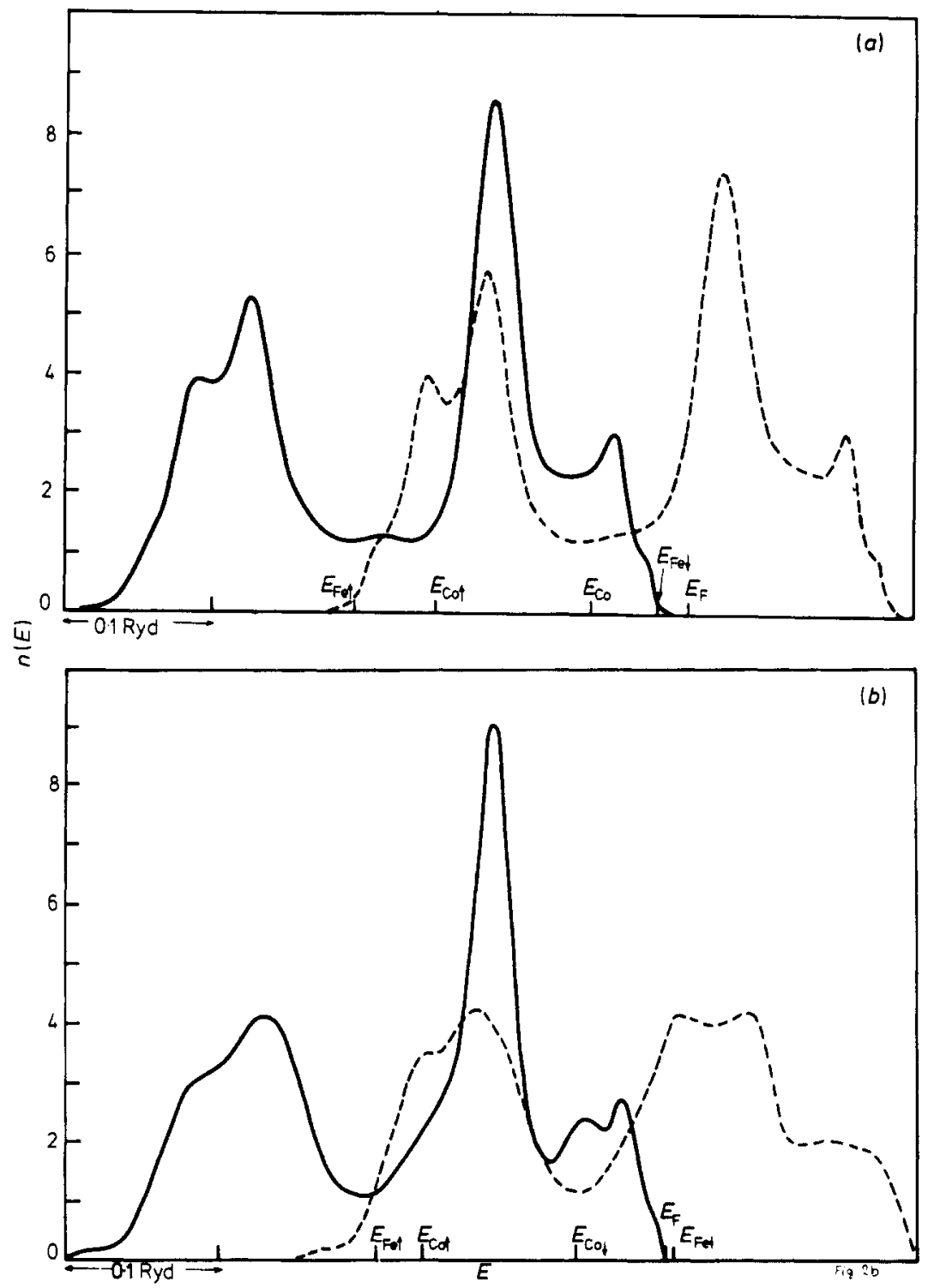

Figure 2. Densities of states of $(a)$ ordered and $(b)$ disordered ferromagnetic FeCo: broken curves, $n_{\downarrow}(E)$; full curves, $n_{1}(E)$. 
spin band, the difference $\Delta E_{\downarrow}=E_{\mathrm{Fe} \downarrow}-E_{\mathrm{C} 。 \downarrow}$ between the effective atomic levels is always positive and larger in the disordered case. So, the second peak of iron is pulled up to higher energies while that of cobalt is pulled down to lower energies; the two peaks do not coincide as shown in figure 3 and the total density of states is smooth. For the majority spin bands, the difference $\Delta E_{\uparrow}=E_{\mathrm{Fe} \uparrow}-E_{\mathrm{Co} \uparrow}$ is of opposite sign and only compensates the distance between the two peaks; there is a superposition of the two peaks in both cases. According to simple geometrical reasoning, the ordered state where the number of bonds between atoms of different types is the more numerous leads to a larger charge transfer. In both cases, cobalt with the lower atomic level gets some charge and the charge transfer per cobalt atom is 0.1 electrons in the ordered state and only 0.06 electrons in the disordered state. The magnetic moments deduced agree with the experimental values given by Shull and Wilkinson (1955):

$$
\begin{aligned}
& \left.\begin{array}{l}
M_{\mathrm{Fe}}=3.10 \mu_{\mathrm{B}} \\
M_{\mathrm{Co}_{\mathrm{o}}}=1.60 \mu_{\mathrm{B}}
\end{array}\right\} \quad \text { in the ordered case } \\
& \left.\begin{array}{l}
M_{\mathrm{Fe}}=3.06 \mu_{\mathrm{B}} \\
M_{\mathrm{Co}}=1.64 \mu_{\mathrm{B}}
\end{array}\right\} \quad \text { in the disordered case }
\end{aligned}
$$

Obviously, the values of these moments equal the number of $d$ holes of the component corrected by the charge transfer; the mean value of the magnetic moments does not depend on the order. The values of the electronic specific heat deduced from the density of states of the Fermi level are respectively 2.8 and $3.24 \mathrm{~mJ} \mathrm{~mol}^{-1} \mathrm{~K}^{-2}$ in the ordered and disordered states. They are in good agreement with the experimental results of Kuentzler (1973); as he observes, the electronic specific heat decreases on ordering by nearly $15 \%$. To be sure that the ferromagnetic solution is more stable at low temperatures, we have to compare its energy to the energy of the corresponding paramagnetic phase. Calculations on the paramagnetic state are made by the same

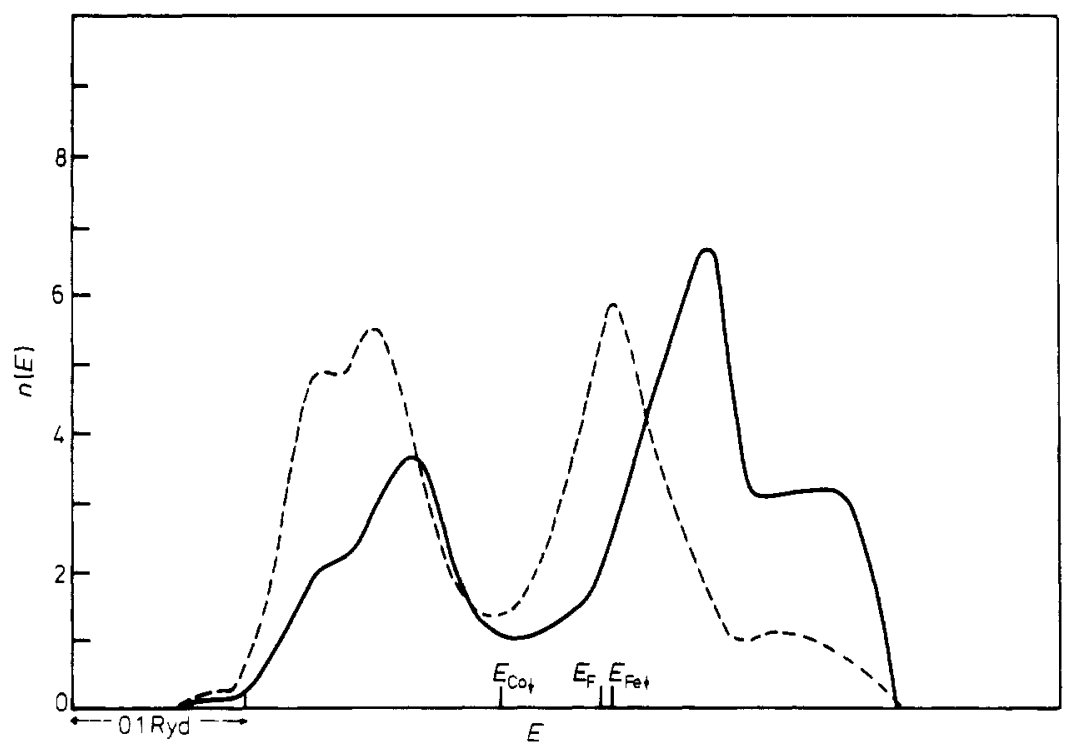

Figure 3. Local densities of states of disordered ferromagnetic $\mathrm{FeCo}$ for spin-down: broken curve, $n_{\mathrm{Co} \downarrow}(E)$; full curve, $n_{\mathrm{Fe} \downarrow}(E)$. 


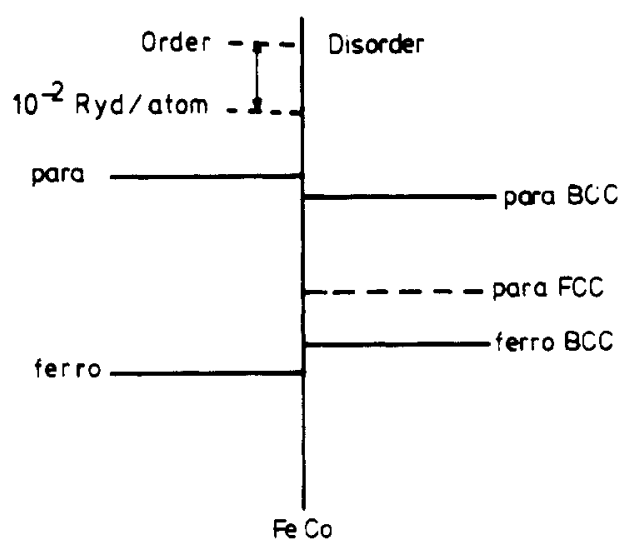

Figure 4. Energy diagram of FeCo.

method. The values of energy deduced from equation (10) are reported in figure 4; they show that for both ordered and disordered states the ferromagnetic phase corresponds to a lower energy than the paramagnetic one. The relative position of the ferromagnetic energies in the ordered and disordered alloys accounts quite well for the existence of an ordered phase at low temperatures. The value of the ordering energy is of the same order of magnitude as that derived from the order-disorder temperatures using Bragg-Williams or Bethe theories (Muto and Takagi 1956). To attempt to explain why the magnetic transition at $1253 \mathrm{~K}$ occurs simultaneously with the structure transition from the BCC to FCC phase, we propose to compare the energy in the disordered paramagnetic BCC and FCC structures. We assume there is no appreciable variation of the atomic volume (which is effectively realised in pure iron for the same transition). The disordered paramagnetic FCC phase is energetically favoured (figure 4), the energy difference between the disordered BCC ferromagnetic and FCC paramagnetic states is of the same order of magnitude as the experimental latent heat found by Orehotsky and Schröder (1974). The paramagnetic FCC density of states is given in figure 5 .

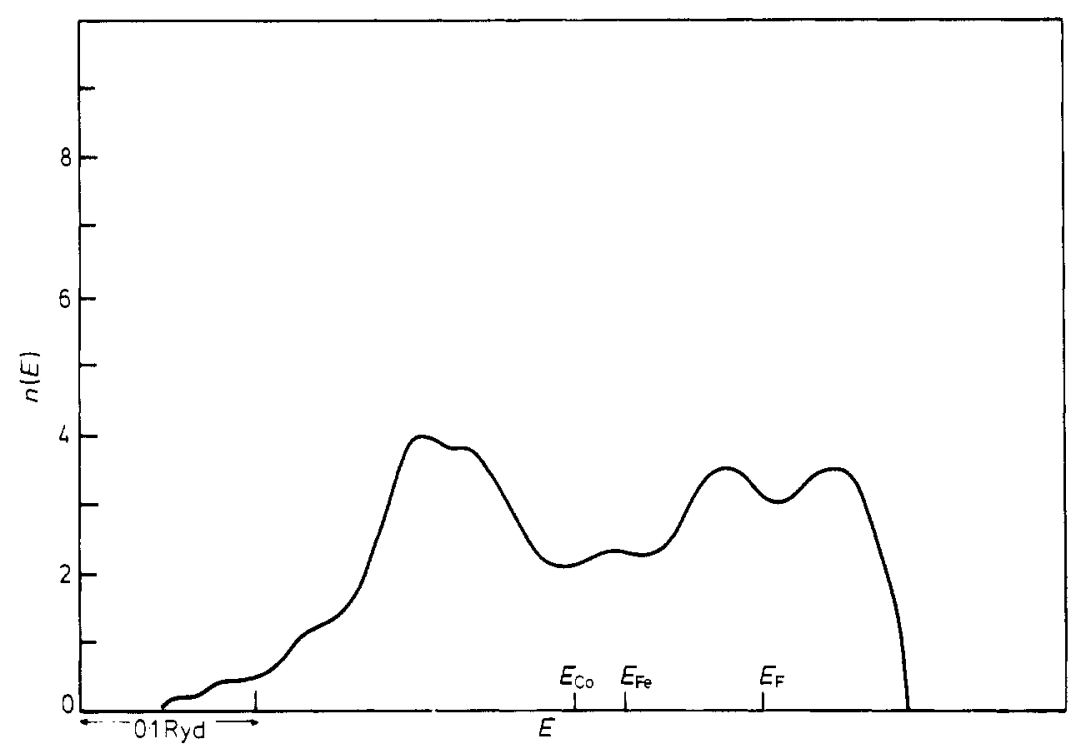

Figure 5. Density of states of disordered paramagnetic FCC FeCo. 


\section{2. $\mathrm{Ni}_{3} \mathrm{Fe}$}

We deduce the values of the hopping integrals from those given by Desjonquères and Cyrot-Lackmann (1975) in the case of FCC nickel with a bandwidth of 0.47 $\mathrm{Ryd}$ for $\mathrm{Fe}$ and $0.25 \mathrm{Ryd}$ for Ni. These values take account of the variation of interatomic distances and structures in the compound compared with the pure metal. The information on nickel is drawn from the recent synchrotron results of Eastman et al (1978). The difference $\left(E_{\mathrm{Fe}}^{0}-E_{\mathrm{Ni}}^{0}\right)$ between the atomic levels of the two components is taken to be 0.105 Ryd (Herman and Skillman 1963). Each atom of $\mathrm{Fe}$ and $\mathrm{Ni}$ brings respectively 7 and 9.3 electrons to the $\mathrm{d}$ band. The value of the Coulomb integral is taken to be $0.33 \mathrm{Ryd}$.

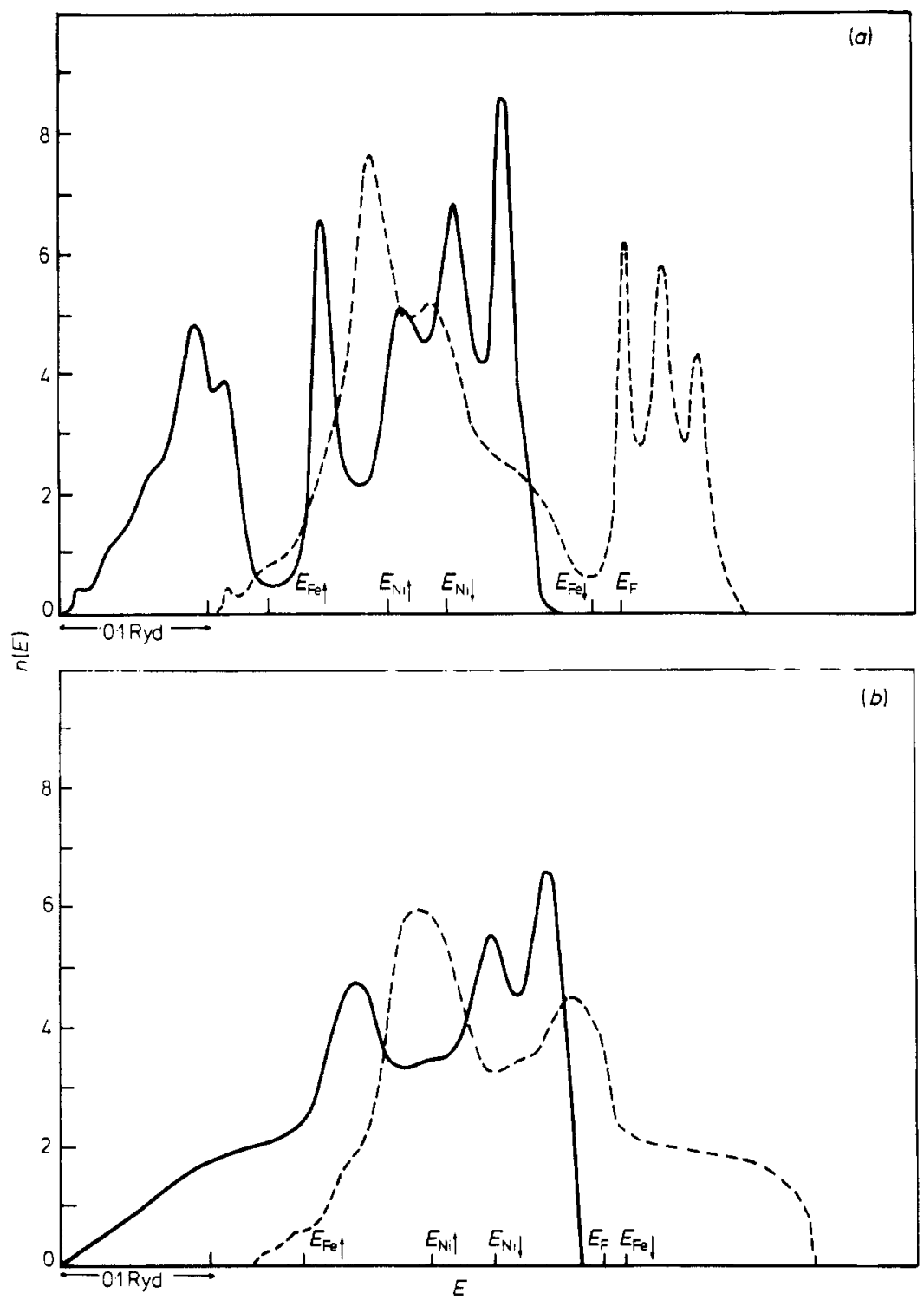

Figure 6. Densities of states of $(a)$ ordered and $(b)$ disordered ferromagnetic $\mathrm{Ni}_{3} \mathrm{Fe}$ : broken curves, $n_{.}(E)$; full curves, $n_{\downarrow}(E)$. 
The self-consistent calculation of the density of states in the ordered and disordered ferromagnetic phases leads in both cases to strong ferromagnetism. Curves are given in figure 6 . The total density of states for each spin exhibits a splitting between two regions with characteristics of either iron or nickel related to the magnitude of the corresponding local density of states. This effect is more pronounced in the ordered case where a small valley appears inside the band corresponding to the narrowing of the two local bands. This can easily be explained; each atom of $\mathrm{Fe}$ has respectively $12 \mathrm{Ni}$ neighbours in the ordered state and only 9 in the average configuration of the disordered state, and each atom of $\mathrm{Ni}$ has respectively 4 and 3 Fe neighbours. So each atom tends to behave as an impurity in the ordered crystal and has a narrower $d$ band. This effect is enhanced in the case of iron by the fact that the ordered state exhibits only $\mathrm{Fe}-\mathrm{Ni}$ bonds with lower hopping integrals compared to $\mathrm{Fe}-\mathrm{Fe}$. All the results are compared with experiments on disordered samples quenched from temperatures high enough to be sure that there is no effect of short-range order. The charge transfer is not very different in the ordered and disordered states, contrary to FeCo, and is about 0.08 electrons per atom of nickel, which has the lower atomic level. The little dependence of the charge transfer with the order in $\mathrm{Ni}_{3} \mathrm{Fe}$ is probably due to the fact that the average disordered surrounding is not so different from the ordered one as for FeCo. The values of the magnetic moment do not depend much on the order, and are in both cases

$$
M_{\mathrm{Fe}}=3.25 \mu_{\mathrm{B}}, \quad M_{\mathrm{Ni}}=0.62 \mu_{\mathrm{B}} .
$$

They are in good agreement with experimental results (Nishi et al 1974). The electronic specific heat $\gamma$ deduced from the density of states at the Fermi level is $3 \mathrm{~mJ}$ $\mathrm{mol}^{-1} \mathrm{~K}^{-2}$ in the disordered phase instead of 3.88 given by experimental results (Y Calvayrac 1978, private communication). It is not possible to compare this value to that of the ordered phase; the position of the Fermi level inside an enhanced peak renders $n\left(E_{\mathrm{F}}\right)$ too sensitive to the choice of parameters. The ferromagnetic phase is more stable at low temperatures than the corresponding paramagnetic phase; furthermore, when the alloy is magnetic, the ordered state is more stable than the disordered one in accordance with the experimental observations (figure 7). We find the right order of succession of phases with increasing temperatures except for high temperatures; but we must remember that the energies of figure 7 are obtained at zero temperature and the introduction of entropic terms will probably restore the

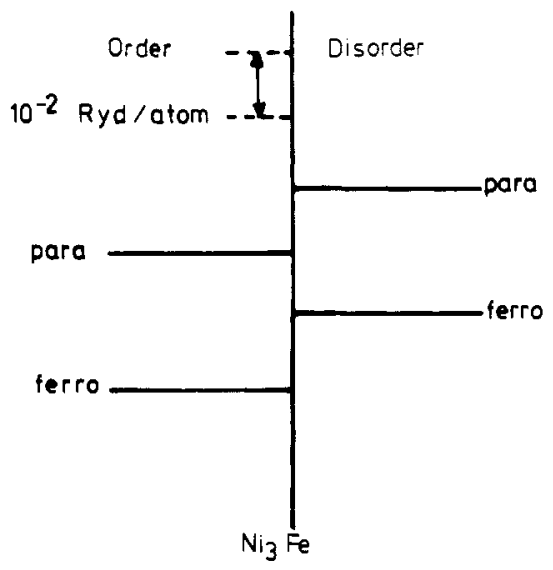

Figure 7. Energy diagram of $\mathrm{Ni}_{3} \mathrm{Fe}$. 


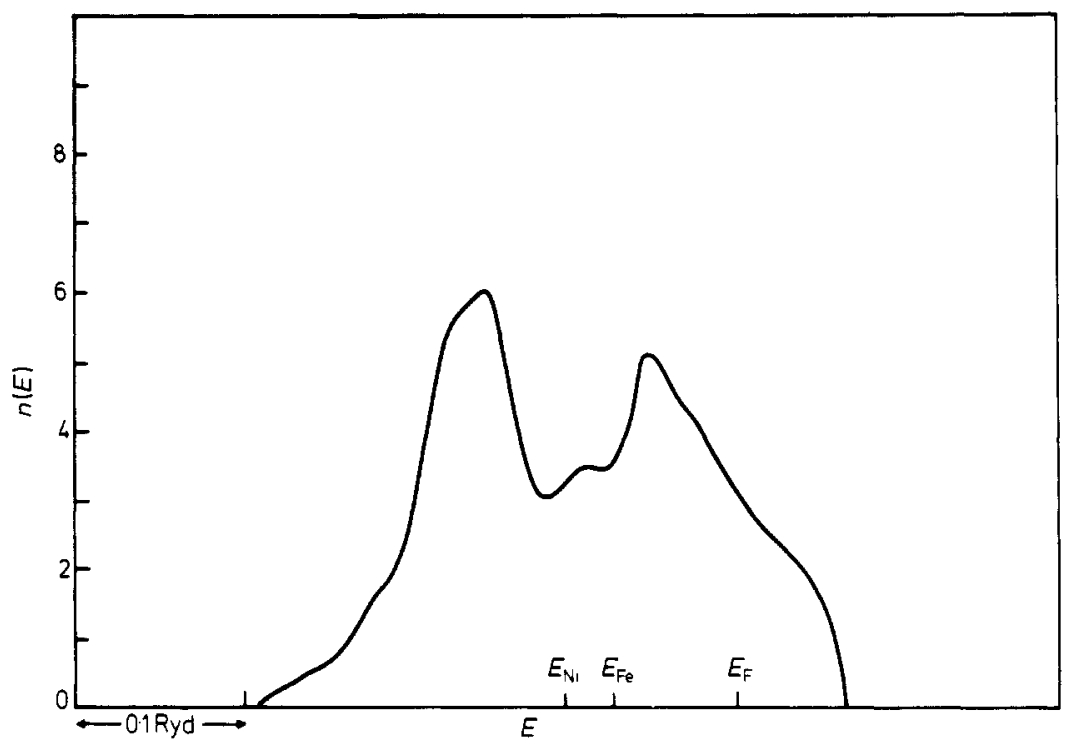

Figure 8. Density of states of disordered paramagnetic $\mathrm{Ni}_{3} \mathrm{Fe}$.

right succession of phases. Figure 8 shows the density of states of the paramagnetic disordered FCC phase. Because of short-range order, we have not studied $\mathrm{Ni}_{3} \mathrm{Fe}$ in the temperature range (776-871 K). Indeed, in order to include its effect, it is necessary to go beyond the single-site CPA approximation. This has already been done for paramagnetic $\mathrm{Ni}_{3} \mathrm{Fe}$ (Cyrot et al 1977), and taking into account ferromagnetism would lead to very tedious calculations.

\section{Conclusion}

In conclusion, we have shown that a rigid band splitting fails to describe the ferromagnetism of alloys, especially when the local magnetic moments of the two components are very different. We have proposed a self-consistent method to determine the spin-up and spin-down densities of states, which could also be used in the case of weak ferromagnetism. The application to ordered and disordered $\mathrm{FeCo}$ and $\mathrm{Ni}_{3} \mathrm{Fe}$ alloys has shown that, contrary to the density of states which varies with order, the magnetic properties are quite insensitive to order, in agreement with experiments. From energetic considerations, our results account well for the successive phase transformations with increasing temperatures.

\section{Acknowledgments}

We would like to thank $M$ Cyrot for his suggestions and his constant interest in this study. We are grateful to C Lacroix-Lyon-Caen and $\mathrm{P}$ Villemain for helpful discussions and to the DGRST for financial support. 


\section{References}

Cyrot M, Cyrot-Lackmann F, Desjonquères M C and Gaspard J P 1977 J. Physique C7 285-8

Desjonquères M C and Cyrot-Lackmann F 1975 J. Phys. F: Metal Phys. 5 1368-84

-1977 J. Phys. F: Metal Phys. 7 61-73

Ducastelle F and Gautier F 1976 J. Phys. F: Metal Phys. 6 2039-62

Eastman D E, Himpsel F J and Knapp J A 1978 Phys. Rev. Lett. 40 1514-7

Gaspard J P and Cyrot-Lackmann F 1973 J. Phys. C: Solid St. Phys. 6 3077-96

Giner J, Brouers F, Gautier F and van der Rest J 1976 J. Phys. F: Metal Phys. 6 1281-96

Hasegawa H and Kanamori J 1971 J. Phys. Soc. Japan 31 382-93

- 1972a J. Phys. Soc. Japan 33 1599-606

-1972b J. Phys. Soc. Japan 33 1607-14

Herman F and Skillman S 1963 Atomic Structure Calculations (New York: Prentice Hall)

Jo T 1976 J. Phys. Soc. Japan $40715-22$

Kuentzler R 1973 Phys. Stat. Solidi b58 519-22

Lavagna M 1978 Thesis Grenoble

Muto T and Takagi Y 1956 The theory of Order-Disorder Transitions in Alloys (New York: Academic Press)

Nishi M, Nakai Y and Kunitomi N 1974 J. Phys. Soc. Japan 37570

Orehotsky J and Schröder K 1974 J. Phys. F: Metal Phys. 4 196-201

Shiba H 1971 Prog. Theor. Phys. 46 77-94

Schull C G and Wilkinson M K 1955 Phys. Rev. 97 304-10

Slater J C and Koster G F 1954 Phys. Rev. 94 1498-524

Yamashita J, Asano S and Hayakawa H 1966a J. Phys. Soc. Japan 21 1323-31

Yamashita J, Wakoh S and Asano S 1966b J. Phys. Soc. Japan 21 53-61 